\title{
Automatic Path Planning and Control Design for Autonomous Landing of UAVs using Dynamic Inversion
}

\author{
Shashiprakash Singh and Radhakant Padhi
}

\begin{abstract}
In this paper a nonlinear control has been designed using the dynamic inversion approach for automatic landing of unmanned aerial vehicles (UAVs), along with associated path planning. This is a difficult problem because of light weight of UAVs and strong coupling between longitudinal and lateral modes. The landing maneuver of the UAV is divided into approach, glideslope and flare. In the approach UAV aligns with the centerline of the runway by heading angle correction. In glideslope and flare the UAV follows straight line and exponential curves respectively in the pitch plane with no lateral deviations. The glideslope and flare path are scheduled as a function of approach distance from runway. The trajectory parameters are calculated such that the sink rate at touchdown remains within specified bounds. It is also ensured that the transition from the glideslope to flare path is smooth by ensuring $C^{1}$ continuity at the transition. In the outer loop, the roll rate command is generated by assuring a coordinated turn in the alignment segment and by assuring zero bank angle in the glideslope and flare segments. The pitch rate command is generated from the error in altitude to control the deviations from the landing trajectory. The yaw rate command is generated from the required heading correction. In the inner loop, the aileron, elevator and rudder deflections are computed together to track the required body rate commands. Moreover, it is also ensured that the forward velocity of the UAV at the touch down remains close to a desired value by manipulating the thrust of the vehicle. A nonlinear six-DOF model, which has been developed from extensive wind-tunnel testing, is used both for control design as well as to validate it.
\end{abstract}

\section{INTRODUCTION}

The capabilities of UAV as flying machines can be exploited to carry out surveillance missions and remote operations. The recovery of UAVs in landing is one of the key operations in flight which define the overall success of the mission. Landing becomes a more challenging task in case of light weight vehicles such as UAVs, mainly because of of the fact that the longitudinal and lateral modes in UAVs are coupled. This problem cannot be dealt effectively with linearized models, where underlying assumption is that the longitudinal and lateral modes are decoupled. In this paper a nonlinear control design approach is proposed using dynamic inversion for automatic landing of UAVs.

Landing trajectories of aerial vehicles typically consists of approach, glideslope and flare [1]. A successful landing would depend upon the good selection of landing trajectory and closely following it. Various methods have been adapted to tackle the problem of automatic landing both for manned

Shashiprakash Singh, Graduate Student, is with Department of Aerospace Engineering, Indian Institute of Science, Bangalore 560012, India.

Radhakant Padhi (Contact author), is Asst. Professor at Department of Aerospace Engineering, Indian Institute of Science, Bangalore 560012, India padhidaero.iisc.ernet.in and unmanned aircrafts. Linearized model of the aircraft have been used in the literature for autolanding using separate longitudinal and lateral dynamics, where the control is designed using linear matrix inequality method [2]. Modern control methods like $\mathrm{H}_{2} / \mathrm{H}_{\infty}$ have also been used for landing of UAVs [3]. However, linear system based approaches have a strong limitation that they work within a small operating range. Gain scheduling can perhaps be used to overcome this limitation to a limited extent. However gain scheduling is a tedious process and there is no guarantee that the interpolated gains can assure stability of the closed loop system [4].

In this paper a nonlinear control has been designed using the dynamic inversion technique [5] for automatic landing of UAVs. Dynamic inversion relies on the philosophy of feedback linearization. This feedback control structure cancels the nonlinearities in the plant such that the closed loop plant behaves like a stable linear system. This method has several advantages, like simplicity in the control structure, ease of implementation, global exponential stability of the tracking error etc. Note that a feedback linearization technique has been successfully demonstrated through simulation for automatic landing of a high performance aircraft [1]. The control implemented for the UAV in this paper has inner loop and outer loop structure. The outer loop converts the guidance commands to body rates. Where as inner loop generates the control to track the body rates desired by outer loop. There is a separate loop for velocity control which regulates the desired velocity by throttle control.

The landing maneuver of the UAV is divided into approach, glideslope and flare. The requirement of variable gain and blending function [6] for gains at the transition of glideslope and flare have been reported in literature. This requirement has been overcome by careful selection of landing trajectory parameters. This ensures that the transition from glideslope to flare path is smooth. The glideslope and flare path parameters are computed online (i.e. they are not fixed apriori). Note that the trajectory parameters are calculated such that the sink rate at touchdown remains within specified bounds.

The proposed control design has been experimented with respect to a UAV built in the UAV lab of Indian Institute of Science. Complete nonlinear six degree of freedom (sixDOF) equations of motion are used to define the dynamics of the UAV. The aerodynamic coefficients for force and moments are found from the curve fitting of the wind tunnel data [7], the philosophy of which is available in the literature [8]. Note that while curve fitting the nonlinearities in the aerodynamic coefficients are not neglected. 


\section{Mathematical Model}

Simulation requires an accurate mathematical model of the dynamical system so that analysis be meaningful. Here nonlinear six-DOF equations are used for the simulation. The aerodynamic force and moment coefficients are found from curve fitting on wind tunnel data of the UAV.

\section{A. Six-DOF equations of motion}

Under the assumptions of airplane to be a rigid body and earth to be flat the complete set of six-DOF equations [9] of motion are given by following differential equations.

$$
\begin{aligned}
\dot{U} & =R V-Q W-g \sin \theta+X_{a}+X_{t} \\
\dot{V} & =P W-R U+g \sin \phi \cos \theta+Y_{a} \\
\dot{W} & =Q U-P V+g \cos \phi \cos \theta+Z_{a} \\
\dot{P} & =c_{1} R Q+c_{2} P Q+c_{3} L_{a}+c_{4} N_{a} \\
\dot{Q} & =c_{5} P R+c_{6}\left(P^{2}-R^{2}\right)+c_{7}\left(M_{a}+M_{t}\right) \\
\dot{R} & =c_{8} P Q-c_{2} R Q+c_{4} L_{a}+c_{9} N_{a} \\
\dot{\phi} & =P+Q \sin \phi \tan \theta+R \cos \phi \tan \theta \\
\dot{\theta} & =Q \cos \phi-R \sin \phi \\
\dot{\psi} & =Q \sin \phi \sec \theta+R \cos \phi \sec \theta \\
\dot{h} & =U \sin \theta-V \sin \phi \cos \theta-W \cos \phi \cos \theta
\end{aligned}
$$

In above equations $U, V, W$ are velocity components and $P, Q, R$ are roll, pitch and yaw rates respectively about the body axis. $\phi, \theta, \psi$ are euler angles and $h$ is the height above ground. $\mathrm{x}$ is forward distance and $\mathrm{y}$ is sideward distance. $\mathrm{x}$ and $\mathrm{y}$ position equations are not written above, but they are included in the simulation. $X_{a}, Y_{a}, Z_{a}$ are the aerodynamic forces per unit mass and $L_{a}, M_{a}, N_{a}$ are the aerodynamic moments about the body axis. $X_{t}$ is the force per unit mass in direction $X$ due to thrust and $M_{t}$ is the moment around the $Y$ axis caused by thrust offset from the center of gravity (CG) of the aircraft.

\section{B. Aerodynamic forces and moments}

The aerodynamic forces and moments are given by

$$
\begin{aligned}
{\left[\begin{array}{lll}
X_{a} & Y_{a} & Z_{a}
\end{array}\right] } & =\frac{\bar{q} S}{m}\left[\begin{array}{lll}
C_{X} & C_{Y} & C_{Z}
\end{array}\right] \\
{\left[\begin{array}{lll}
L_{a} & M_{a} & N_{a}
\end{array}\right] } & =\bar{q} S\left[\begin{array}{lll}
b C_{l} & c C_{m} & b C_{n}
\end{array}\right] \\
X_{t} & =\frac{1}{m}\left(T_{\max } \sigma_{t}\right) \\
M_{t} & =-d\left(T_{\max } \sigma_{t}\right)
\end{aligned}
$$

where $\bar{q}$ is dynamic pressure and $S$ is wing planform area. $b$ is wing span and $c$ is chord length. $T_{\max }$ is the maximum thrust $(15 \mathrm{~N})$ which can be produced by the electric motor and propeller assembly. $\sigma_{t}$ is throttle control which varies from 0 to 1 . It is assumed that thrust produced has linear relation with throttle input. $d$ is offset of the thrust line from the $\mathrm{CG}$ of the vehicle.
Aerodynamic coefficients obtained from curve fitting on wind tunnel data [7] are given as

$C_{X}=C_{X_{0}}+C_{X_{\alpha}}(\alpha) \alpha+C_{X_{\delta e}}(\alpha) \delta e+C_{X_{Q}}(\alpha) \bar{Q}$

$C_{Y}=C_{Y_{\beta}}(\alpha) \beta+C_{Y_{\delta a}}(\alpha) \delta a+C_{Y_{\delta r}}(\alpha) \delta r+C_{Y_{P}}(\alpha) \bar{P}+C_{Y_{R}}(\alpha) \bar{R}$

$C_{Z}=C_{Z_{0}}+C_{Z_{\alpha}}(\alpha) \alpha+C_{Z_{\beta}} \beta+C_{Z_{\delta e}} \delta e+C_{Z_{Q}}(\alpha) \bar{Q}$

$C_{l}=C_{l_{\beta}}(\alpha) \beta+C_{l_{\delta a}}(\alpha) \delta a+C_{l_{P}}(\alpha) \bar{P}+C_{l_{R}}(\alpha) \bar{R}$

$C_{m}=C_{m_{0}}+C_{m_{\alpha}}(\alpha) \alpha+C_{m_{\beta}}(\alpha, \beta) \beta+C_{m_{\delta e}}(\alpha) \delta e+C_{m_{Q}}(\alpha) \bar{Q}$

$C_{n}=C_{n_{\beta}}(\alpha) \beta+C_{n_{\delta r}}(\alpha) \delta r+C_{n_{P}}(\alpha) \bar{P}+C_{n_{R}}(\alpha) \bar{R}$

where,

$$
\left[\begin{array}{lll}
\bar{P} & \bar{Q} & \bar{R}
\end{array}\right]=\frac{1}{2 V_{t}}\left[\begin{array}{lll}
b P & c Q & b R
\end{array}\right]
$$

In above equations $\alpha$ is angle of attack and $\beta$ is sideslip angle. $\delta a, \delta e, \delta r$ are aileron elevator and rudder control deflections respectively. Some of the static and dynamic derivatives are functions of $\alpha$ and $\beta$. The detailed functions and constants are given in appendix. Only static tests were conducted in wind tunnel. The dynamic derivatives are found with Athennae Vortex Lattice (AVL) software [10].

\section{The UAV}

Numerical simulations are carried out with the data of a prototype UAV, named as all electric airplane-2 (AE-2) [7]. It is designed and developed at UAV lab of aerospace engineering department, IISc. AE-2 (Fig. 1) is a fixed wing airplane designed for autonomous flying. It has been designed for long endurance, where the flight duration is expected to be around 45 minutes. The thrust generating unit of the AE2 is an electric motor with propeller, which is powered by lithium-polymer battery. It has a pusher configuration of thrust, this has been done so that gimballed camera can be mounted at the nose. The physical data of AE-2 is given in Table I.

TABLE I

PHYSICAL DATA OF AE-2

\begin{tabular}{|c|c|c|c|c|c|c|}
\hline$b$ & $c$ & $m$ & $I_{x x}$ & $I_{y y}$ & $I_{z z}$ & $\begin{array}{c}I_{x z} \\
k^{2}\end{array}$ \\
$m$ & $k g$ & $k^{2} m^{2}$ & $k^{2} m^{2}$ & kgm $^{2}$ & kgm $^{2}$ \\
\hline 2 & 0.3 & 6 & 0.5062 & 0.89 & 0.91 & 0.0015 \\
\hline
\end{tabular}

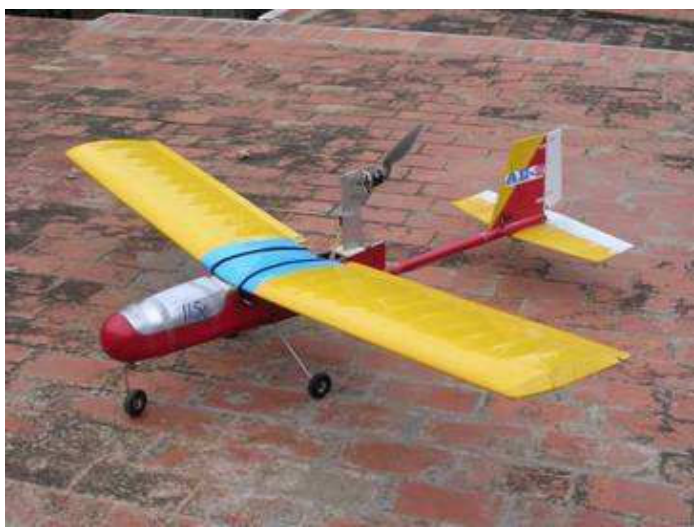

Fig. 1. AE-2 (All Electric airplane-2) 


\section{Path Planning and Guidance}

The autonomous landing of UAVs requires a good path planning and guidance. Here the landing is divided into three phases, which are approach, glideslope and flare. The desired trajectory is made a function of forward distance.

\section{A. Approach}

During approach the vehicle should come and align with the runway at a specified height from where the glideslope can be started.

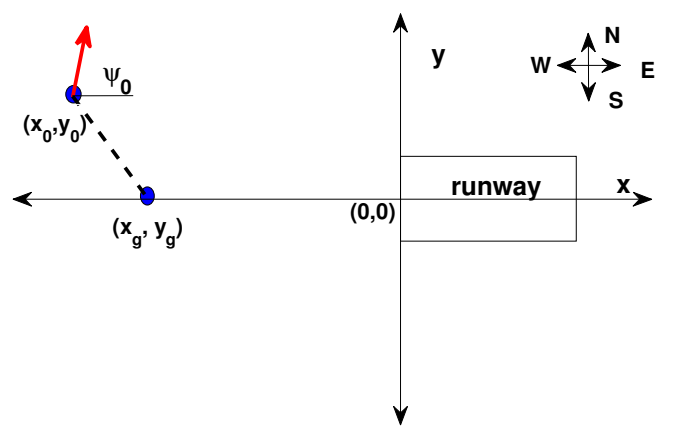

Fig. 2. Approach geometry in $x-y$ plane

In Fig. $2\left(x_{0}, y_{0}\right)$ is the initial point where UAV is at the beginning of landing command. $\left(x_{g}, y_{g}=0\right)$ is point from where glideslope begins. $\psi_{0}$ is the initial orientation of the vehicle. The equation for path of approach can be written as a straight line function of forward distance.

$$
y^{*}=y_{0}+\left(\frac{y_{g}-y_{0}}{x_{g}-x_{0}}\right)\left(x-x_{0}\right)
$$

We can find the desired heading angle to be followed by inverting the kinematics. For this we first write a first order error differential equation for $\mathrm{y}$, where error $e_{y}=y-y^{*}$

$$
\dot{e_{y}}+k_{y} e_{y}=0
$$

The solution of the above differential equation is given as $e_{y}=e_{y_{0}} e^{-k_{y} t}$. If $k_{y}$ is chosen positive than $e_{y} \rightarrow 0$ as $t \rightarrow \infty$. Expanding (16) we can write,

$$
\begin{gathered}
\left(\dot{y}-\dot{y}^{*}\right)+k_{y}\left(y-y^{*}\right)=0 \\
\dot{y}=\dot{y}^{*}-k_{y}\left(y-y^{*}\right)
\end{gathered}
$$

From six-DOF equations we can write,

$$
\dot{y}=a_{y} \sin \psi+b_{y} \cos \psi
$$

where,

$$
\begin{aligned}
& a_{y} \triangleq U \cos \theta+V \sin \phi \sin \theta+W \cos \phi \sin \theta \\
& b_{y} \triangleq V \cos \phi-W \sin \phi
\end{aligned}
$$

Substituting (19) in (18) than solving for $\psi$

$$
\psi^{*}=\sin ^{-1}\left(\frac{\dot{y}^{*}-k_{y}\left(y-y^{*}\right)}{\sqrt{a_{y}^{2}+b_{y}^{2}}}\right)-\tan ^{-1}\left(\frac{b_{y}}{a_{y}}\right)
$$

\section{B. Glideslope}

During glideslope the height of the vehicle above ground is controlled. The desired height is scheduled as a function of forward distance. The glideslope is a straight line path whose slope is defined by the flight path angle.

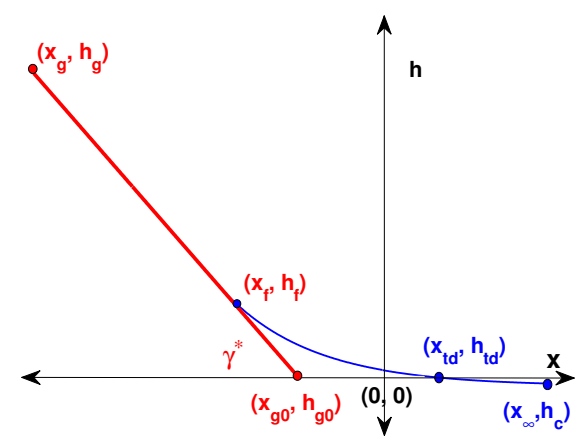

Fig. 3. Landing geometry in $\mathrm{x}-\mathrm{h}$ plane

In Fig. $3\left(x_{g}, h_{g}\right)$ is the point where glideslope begins. $\left(x_{f}, h_{f}\right)$ is point where flare begins. $\left(x_{t d}, h_{t d}=0\right)$ is the touchdown point. $(0,0)$ is the origin of inertial frame at the beginning of runway. $\left(x_{g_{0}}, h_{g_{0}}=0\right)$ is a fictitious point on ground from where glideslope is projected. $\left(x_{\infty}, h_{c}\right)$ is the final point of flare path, this point is chosen to be below ground so that exponential flare path intersects the ground at touchdown point.

When the UAV enters in glideslope initiation zone the required flight path angle which vehicle has to follow is calculated as follows

$$
\gamma^{*}=\tan ^{-1}\left(\frac{h_{g}-h_{g_{0}}}{x_{g}-x_{g_{0}}}\right)
$$

Than the desired height at every point of glideslope can be written as a function of forward distance

$$
h^{*}=\left(x-x_{g_{0}}\right) \tan \gamma^{*}
$$

\section{Flare}

During flare the height of the vehicle above ground is controlled. The flare path is an exponential curve. The desired height during exponential flare can be scheduled as a function of forward distance.

$$
h^{*}=h_{c}+\left(h_{f}-h_{c}\right) e^{-k_{x}\left(x-x_{f}\right)}
$$

The unknowns in above equation are flare height $h_{f}$, distance at which to begin flare $x_{f}$, final height below the ground where flare trajectory should end $h_{c}$, and constant $k_{x}$. We can solve for these four unknowns under the following four constraints,

Initial condition: The point where glideslope ends and flare begins be coincident. Substituting $x=x_{f}$ in (22) and (23) than equating

$$
h_{f}=-\left(x_{f}-x_{g_{0}}\right) \tan \gamma^{*}
$$


Initial slope: The slope at the beginning of flare and at the end of glideslope be same. Differentiating (22) and (23) than equating at $x=x_{f}$

$$
\left(h_{f}-h_{c}\right) k_{x}=\tan \gamma^{*}
$$

Touchdown condition: The flare trajectory should intersect the ground at touchdown point. Replacing $x=x_{t d}$ and $h^{*}=0$ in (23) we get,

$$
0=h_{c}+\left(h_{f}-h_{c}\right) e^{-k_{x}\left(x_{t d}-x_{f}\right)}
$$

Sink rate at touchdown: The descent rate at touchdown should be equal to specified sink rate. Differentiating (23) and evaluating at $x=x_{t d}$. Putting $\dot{h}_{*}=\dot{h}_{t d}^{*}$, where $\dot{h}_{t d}^{*}$ is the desired sink rate at touchdown. Here we assume the forward velocity at touchdown is nearly equal to air velocity.

$$
\dot{h}_{t}^{*}=-\left(h_{f}-h_{c}\right) k_{x} \dot{x}_{t d} e^{-k_{x}\left(x_{t d}-x_{f}\right)}
$$

Now we can solve the (24-27) for the four unknowns of the landing trajectory. The solution will ensure a smooth transition from glidelsope to flare path. We also have the direct control over the touchdown point and sink rate at touchdown. Which can be the design parameters and tuned as per the need. Now the goal of control design is to closely track the desired landing trajectory.

\section{Control Design}

Dynamic Inversion is a promising technique for nonlinear control design based on theory of feedback linearization. In dynamic inversion the nonlinearities are canceled by feedback so that linear control system theory may be applied. This is achieved by enforcing stable error dynamics so that error goes towards zero by time hence achieve the tracking.

Here the control is divide into outer and inner loop. The outer loop transforms the guidance commands into the body rates. Further inner loop generates the control required for tacking the desired body rates commanded by outer loop.

\section{A. Outer Loop Control}

1) Heading control: The heading is controlled during landing by generating yaw rate command from the error in heading angle. The desired heading is found from the lateral deviation from runway. Writing first order error dynamics for $\psi$, where error $=\psi-\psi^{*}$

$$
\begin{gathered}
\left(\dot{\psi}-\dot{\psi}^{*}\right)+k_{\psi}\left(\psi-\psi^{*}\right)=0 \\
\dot{\psi}=\dot{\psi}^{*}-k_{\psi}\left(\psi-\psi^{*}\right)
\end{gathered}
$$

Separating terms containing $R$ from $\dot{\psi}$ equation (9) and rearranging above equation we get,

$$
R^{*}=\sec \phi \cos \theta\left(\dot{\psi}^{*}-k_{\psi}\left(\psi-\psi^{*}\right)\right)-Q \tan \phi
$$

2) Altitude control: The height during landing is controlled by generating pitch command from the error in height. The desired height is found from the guidance. Writing second order error dynamics for $h$, where error $=h-h^{*}$

$$
\begin{gathered}
\left(\ddot{h}-\ddot{h^{*}}\right)+k_{\dot{h}}\left(\dot{h}-\dot{h}^{*}\right)+k_{h}\left(h-h^{*}\right)=0 \\
\ddot{h}=\ddot{h^{*}}-k_{\dot{h}}\left(\dot{h}-\dot{h}^{*}\right)-k_{h}\left(h-h^{*}\right)
\end{gathered}
$$

Under the assumption that the velocities do not change much in one time step, we differentiate $\dot{h}$ equation (10) in order to get $\ddot{h}$. We can write symbolically

$$
\ddot{h}=a_{h}+b_{h} \dot{\phi}+c_{h} \dot{\theta}
$$

where $a_{h}, b_{h}, c_{h}$ are defined as

$$
\begin{aligned}
& a_{h} \triangleq \dot{U} \sin \theta-(\dot{V} \sin \phi+\dot{W} \cos \phi) \cos \theta \\
& b_{h} \triangleq-(V \cos \phi-W \sin \phi) \cos \theta \\
& c_{h} \triangleq U \cos \theta+(V \sin \phi+W \cos \phi) \sin \theta
\end{aligned}
$$

Substituting back equation (33) in (32)

$$
a_{h}+b_{h} \dot{\phi}+c_{h} \dot{\theta}=\ddot{h^{*}}-k_{\dot{h}}\left(\dot{h}-\dot{h}^{*}\right)-k_{h}\left(h-h^{*}\right)
$$

Now separating the terms containing $\mathrm{Q}$, in $\dot{\phi}$ and $\dot{\theta}$ from equation (7) and (8) respectively

$$
\begin{aligned}
& \dot{\phi}=f_{\phi}+g_{\phi} Q \\
& \dot{\theta}=f_{\theta}+g_{\theta} Q
\end{aligned}
$$

where, $\quad f_{\phi} \triangleq P+R \cos \phi \tan \theta \quad g_{\phi} \triangleq \sin \phi \tan \theta$

$$
f_{\theta} \triangleq-R \sin \phi \quad g_{\theta} \triangleq \cos \phi
$$

Substituting (35) and (36) in (34) than rearranging

$$
Q^{*}=\frac{\ddot{h^{*}}-k_{\dot{h}}\left(\dot{h}-\dot{h}^{*}\right)-k_{h}\left(h-h^{*}\right)-\left(a_{h}+b_{h} f_{\phi}+c_{h} f_{\theta}\right)}{\left(b_{h} g_{\phi}+c_{h} g_{\theta}\right)}
$$

3) Bank angle control: To control bank angle roll rate command is generated from the error in roll angle. Where desired roll angle is zero for level flight and in the case of a turn it is found from the coordinated turn constraint. Writing first order error dynamics for $\phi$, where error $=\phi-\phi^{*}$

$$
\begin{gathered}
\left(\dot{\phi}-\dot{\phi}^{*}\right)+k_{\phi}\left(\phi-\phi^{*}\right)=0 \\
\dot{\phi}=\dot{\phi}^{*}-k_{\phi}\left(\phi-\phi^{*}\right)
\end{gathered}
$$

separating terms containing $P$ from $\dot{\phi}$ equation (7) and rearranging above equation we get,

$$
P^{*}=\dot{\phi}^{*}-k_{\phi}\left(\phi-\phi^{*}\right)-(Q \sin \phi+R \cos \phi) \tan \theta
$$

\section{B. Inner Loop Control}

1) Body rate control: To achieve successful landing the control should be calculated such that it tracks the body angular rates desired by the outer guidance loop. We can write objective as $\left[\begin{array}{lll}P & Q & R\end{array}\right]^{T} \longrightarrow\left[\begin{array}{lll}P^{*} & Q^{*} & R^{*}\end{array}\right]^{T}$. Let error be $e=\left[\begin{array}{lll}\left(P-P^{*}\right) & \left(Q-Q^{*}\right) & \left(R-R^{*}\right)\end{array}\right]^{T}$. Writing first order error dynamics 


$$
\begin{gathered}
{\left[\begin{array}{c}
\dot{P}-\dot{P}^{*} \\
\dot{Q}-\dot{Q}^{*} \\
\dot{R}-\dot{R}^{*}
\end{array}\right]+\left[\begin{array}{ccc}
k_{P} & 0 & 0 \\
0 & k_{Q} & 0 \\
0 & 0 & k_{R}
\end{array}\right]\left[\begin{array}{c}
P-P^{*} \\
Q-Q^{*} \\
R-R^{*}
\end{array}\right]=0} \\
{\left[\begin{array}{c}
\dot{P} \\
\dot{Q} \\
\dot{R}
\end{array}\right]=\left[\begin{array}{c}
P^{*}-k_{P}\left(P-P^{*}\right) \\
\dot{Q}^{*}-k_{Q}\left(Q-Q^{*}\right) \\
\dot{R}^{*}-k_{R}\left(R-R^{*}\right)
\end{array}\right]}
\end{gathered}
$$

separating the state and control terms in $\dot{P}, \dot{Q}, \dot{R}$ from equation (4-6) and rearranging above equation we can write

$$
f_{R}+g_{R} U_{c}=b_{R}
$$

carrying out the necessary algebra, the solution is

$$
U_{c}=g_{R}^{-1}\left(b_{R}-f_{R}\right)
$$

where, $U_{c}=\left[\begin{array}{lll}\delta a & \delta e & \delta r\end{array}\right]^{T}$ and other terms are defined as follows

$$
\begin{gathered}
f_{R} \triangleq\left[\begin{array}{c}
c_{1} R Q+c_{2} P Q+c_{3} L_{a_{x}}+c_{4} N_{a_{x}} \\
c_{5} P R+c_{6}\left(P^{2}-R^{2}\right)+c_{7}\left(M_{a_{x}}-M_{t}\right) \\
c_{8} P Q-c_{2} R Q+c_{4} L_{a_{x}}+c_{9} N_{a_{x}}
\end{array}\right] \\
g_{R} \triangleq\left[\begin{array}{ccc}
c_{3} L_{a_{u}} & 0 & c_{4} N_{a_{u}} \\
0 & c_{7} M_{a_{u}} & 0 \\
c_{4} L_{a_{u}} & 0 & c_{9} N_{a_{u}}
\end{array}\right] \\
b_{R} \triangleq\left[\begin{array}{c}
\dot{P^{*}}-k_{P}\left(P-P^{*}\right) \\
\dot{Q}^{*}-k_{Q}\left(Q-Q^{*}\right) \\
R^{*}-k_{R}\left(R-R^{*}\right)
\end{array}\right]
\end{gathered}
$$

where,

$$
\begin{aligned}
& L_{a_{x}} \triangleq \bar{q} S b\left[C_{l_{\beta}}(\alpha) \beta+C_{l_{P}}(\alpha) P+C_{l_{R}}(\alpha) R\right] \\
& M_{a_{x}} \triangleq \bar{q} S c\left[C_{m_{0}}+C_{m_{\alpha}}(\alpha) \alpha+C_{m_{\beta}}(\alpha, \beta) \beta+C_{m_{Q}}(\alpha) Q\right] \\
& N_{a_{x}} \triangleq \bar{q} S b\left[C_{n_{\beta}}(\alpha) \beta+C_{n_{P}}(\alpha) P+C_{n_{R}}(\alpha) R\right] \\
& \text { and, } \\
& L_{a_{u}} \triangleq \bar{q} S b C_{l_{\delta a}} ; \quad M_{a_{u}} \triangleq \bar{q} S c C_{m_{\delta e}} ; \quad N_{a_{u}} \triangleq \bar{q} S b C_{n_{\delta r}}
\end{aligned}
$$

2) Velocity control: The forward velocity is maintained constant during landing by controlling the thrust through throttle. We can write error in velocity as $\left(U-U^{*}\right)$, where $U^{*}$ is the velocity which has to be maintained during landing. Enforcing the first order error dynamics.

$$
\begin{gathered}
\left(\dot{U}-\dot{U}^{*}\right)+k_{U}\left(U-U^{*}\right)=0 \\
\dot{U}=\dot{U}^{*}-k_{U}\left(U-U^{*}\right)
\end{gathered}
$$

Separating the state and control terms in $\dot{U}$ from (1) and rearranging

$$
f_{U}+g_{U} \sigma_{t}=b_{U}
$$

The control solution is

$$
\sigma_{t}=g_{U}^{-1}\left(b_{U}-f_{U}\right)
$$

where,

$$
\begin{aligned}
& f_{U} \triangleq R V-Q W-g \sin \theta+X_{a} \\
& g_{U} \triangleq \frac{T_{\max }}{m} \\
& b_{U} \triangleq \dot{U}^{*}-k_{U}\left(U-U^{*}\right)
\end{aligned}
$$

\section{Control structure}

Fig. 4 is the schematic diagram of the overall control structure. The velocity loop is not shown in figure. Note that desire height $\left(h^{*}\right)$, desires heading angle $\left(\psi^{*}\right)$ and desired roll angle $\left(\phi^{*}\right)$ are coming from guidance.

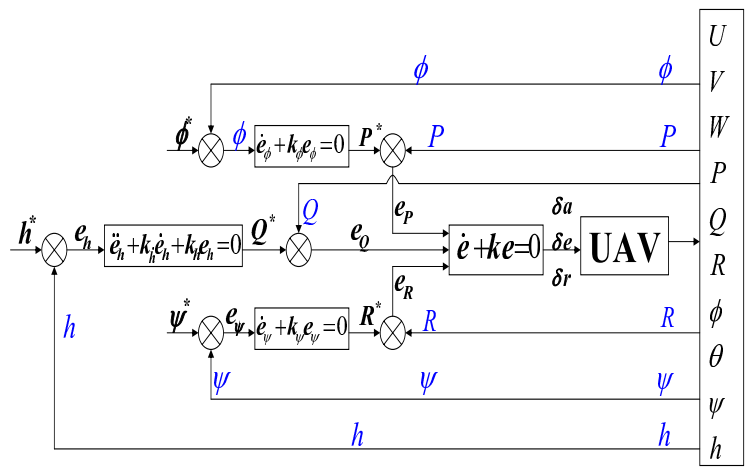

Fig. 4. Control structure

\section{Simulation Results}

To evaluate the algorithm various cases have been simulated with different initial conditions of which two cases are presented here. The simulation results are given along with the result in tabulated form.

\section{A. Landing results}

The complete landing sequence of approach, glideslope and flare has been simulated. Two cases with different initial conditions are considered. One such case (Case 2) is also considered in which the UAV has to loose height during approach. Fig. 5 shows the trajectory followed by the UAV during landing. Initial part of the trajectory is approach where it is aligning with runway. Next part is descent and flare which are in longitudinal plane.

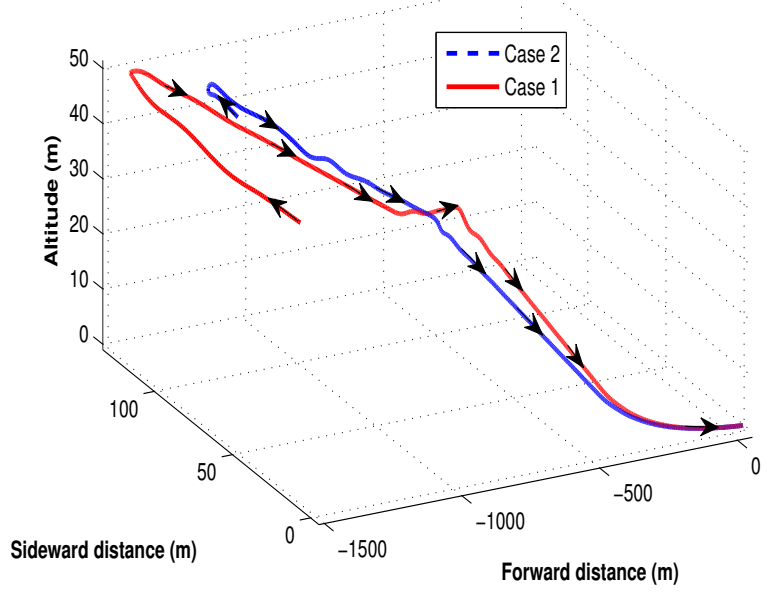

Fig. 5. Trajectory in 3-D during landing 
Fig. 6 shows the landing trajectory in horizontal plane. Where as Fig. 7 shows the landing trajectory in vertical or longitudinal plane. The Fig. 8 shows the control plots for landing. It is observed that in initial part of landing there are some oscillations in control values this is because in initial segment the lateral and longitudinal both the maneuvers are taking place.

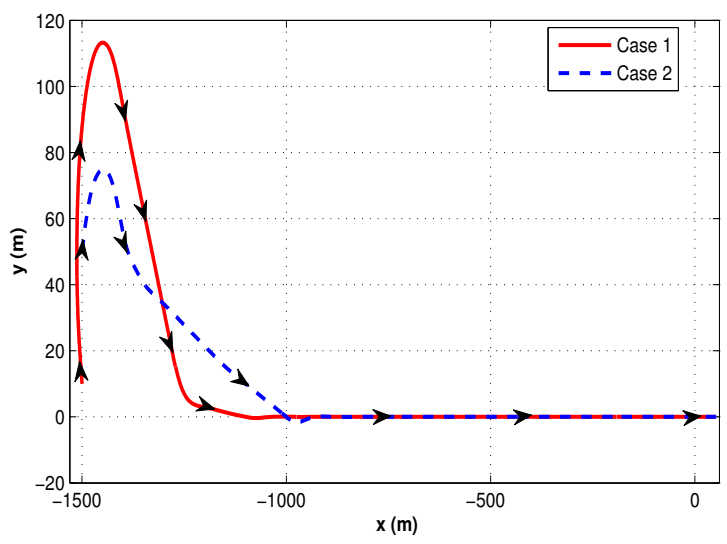

Fig. 6. Trajectory in $x-y$ plane during landing

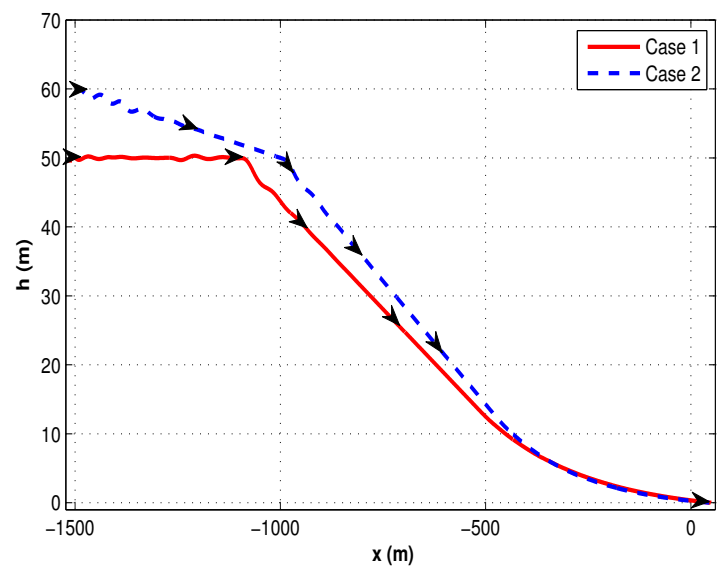

Fig. 7. Trajectory in $\mathrm{x}-\mathrm{h}$ plane during landing
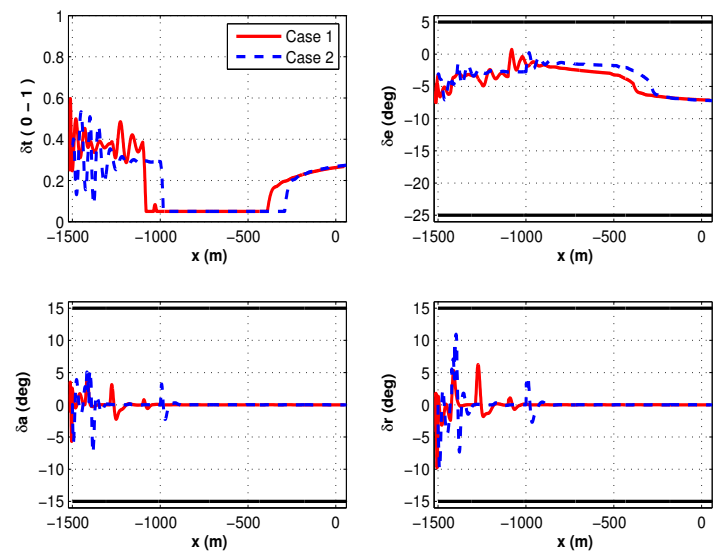

Fig. 8. Throttle, elevator, aileron and rudder controls

The numerical results are given in Table II. It can be observed that the desired touchdown point $(50 \mathrm{~m})$ and desired sink rate $(-0.1 \mathrm{~m} / \mathrm{s})$ at touchdown are achieved successfully.
TABLE II

LANDING RESULTS

\begin{tabular}{|c|c|c|c|c|c|}
\hline & $\begin{array}{c}\left(x_{0}, y_{0}, h_{0}\right) \\
\mathrm{m}\end{array}$ & $\begin{array}{c}\psi_{0} \\
\mathrm{deg}\end{array}$ & $\begin{array}{c}\left(x_{g}, y_{g}\right) \\
\mathrm{m}\end{array}$ & $\begin{array}{c}x_{t d} \\
\mathrm{~m}\end{array}$ & $\begin{array}{c}\dot{h}_{t d} \\
\mathrm{~m} / \mathrm{s}\end{array}$ \\
\hline Case 1 & $(-1500,10,50)$ & $120^{\circ}$ & $(-1000,50)$ & 48.76 & -0.1001 \\
\hline Case 2 & $(-1500,50,60)$ & $45^{\circ}$ & $(-1100,50)$ & 48.97 & -0.1001 \\
\hline
\end{tabular}

\section{Vi. Conclusions}

In this paper a guidance and nonlinear control design for automatic landing has been demonstrated. The simulation results are promising and show the robustness of the algorithm for different initial conditions. The glideslope and flare path parameters are calculated online, such that it achieves the touchdown point. The sink rate at touchdown remains within specified bounds. It is also ensured that the transition from the glideslope to flare is smooth. The dynamic inversion technique is used for nonlinear control design. The underlying assumption of dynamic inversion is that we have a accurate knowledge of the dynamic model and true estimate of the states. However these problems can be addressed by augmenting dynamic inversion with neuroadaptive technique and using extended kalman filter for state estimation. Further algorithm needs to be tested in presence of wind shear and wind gust.

\section{ACKNOWLEDGEMENT}

The authors would like to acknowledge the support of UK-India Education Research Initiative for the project (CSSP/UKIE002) under which this research was funded.

\section{REFERENCES}

[1] B. Prasad and S. Pradeep, "Automatic Landing System Design using Feedback Linearization Method", Proceedings of AIAA Infotech Aerospace Conference and Exhibit, 2007, Rohnert Park, California.

[2] P. Rosa, C. Silvestre, D. Cabenchias and R. Cunha, "Autolanding Controller for a Fixed Wing Unmanned Air Vehicle", AIAA Guidance Navigation and Control Conference and Exhibit, 2007, Hilton head, South Carolina.

[3] R. Wang and Y. Shen, "Flying-Wing UAV Landing Control and Simulation Based on Mixed $\mathrm{H}_{2} / \mathrm{H}_{\infty}$ ", Proceedings of IEEE International Conference on Mechatronics and Automation, 2007, Harbin, China.

[4] J. S. Shamma and M. Athans, "Analysis of Gain Scheduled Control for Nonlinear Plants", IEEE Transactions On Automatic Control, Vol. 35, No. 8, 1990

[5] D. Enns, D. Bugajski, R. Hendrick and G. Stein, "Dynamic inversion: an evolving methodology for flight control design", International Journal of Control, Vol. 59, Issue 1, 1994 , pages 71 - 91.

[6] K. S. Kumar and J.Shanmugam, "Design of Blending function for landing phase of an Unmanned Air Vehicle" Proceedings of AIAA Infotech Aerospace Conference and Exhibit, 2007, Rohnert Park, California.

[7] V. Surendra nath, S. P. Govindaraju, M. S. Bhat and C. S. N. Rao, "Configuration Development of All Electric Mini Airplane", ADE/DRDO Project, 2004, Project Ref. No: ADEO/MAE/VSU/001, Department of Aerospace Engineering, Indian Institute of Science, Bangalore.

[8] E. Morelli, "Nonlinear Aerodynamic Modelling Using Multivariate Orthogonal Functions", Proceedings of American Control Conference, 1998, Philadelphia, Pennsylvania.

[9] B. Stevens and F. Lewis, "Aircraft Control and Simulation $2^{\text {nd }}$ Edition", J. Wiley \& Sons, 2003.

[10] M. Drella and H. Youngren, "Athena Vortex Lattice Analysis 3.26 User Primer", http://web.mit.edu/drela/Public/web/avl/, 2006. 